\title{
Association analysis between adverse drug reactions to cytarabine therapy and single nucleotide polymorphisms in cytarabine metabolic genes in patients with hematopoietic tumor
}

\author{
Hozumi Tashima', Yuka Endo ${ }^{1)}$ Naoto Okada', Shingen Nakamura ${ }^{3)}$ Kumiko Kagawa ${ }^{3)}$, \\ Shiro Fujii ${ }^{3)}$, Hirokazu Miki ${ }^{4)}$, Keisuke Ishizawa ${ }^{25)}$, Masahiro Abe ${ }^{3)}$, Youichi Sato ${ }^{13 *}$
}

1) Department of Pharmaceutical Information Science, Institute of Biomedical Sciences, Tokushima University Graduate School, Tokushima, Japan

2) Department of Pharmacy, Tokushima University Hospital, Tokushima, Japan

3) Department of Hematology, Endocrinology and Metabolism, Institute of Biomedical Sciences, Tokushima University Graduate School, Tokushima, Japan

4) Division of Transfusion Medicine and Cell Therapy, Tokushima University Hospital, Tokushima, Japan

5) Department of Clinical Pharmacology and Therapeutics, Institute of Biomedical Sciences, Tokushima University Graduate School, Tokushima, Japan

\begin{abstract}
:
Purpose: Cytarabine arabinoside (Ara-C) is an anti-metabolite that is commonly used as a therapeutic agent for acute leukemia; however, it can cause adverse drug reactions, such as digestive disorders, rashes, and fever. Therefore, identification of gene markers that can accurately predict the development of adverse drug reactions is useful for selecting effective drugs for therapy. After entering the cells, Ara-C is metabolized to Ara-C triphosphate, which inhibits DNA synthesis and exhibits antitumor activity. Therefore, we conducted an association study between the adverse reactions to cytarabine therapy and single nucleotide polymorphisms (SNPs) in cytarabine metabolic genes.

Methods: Among the patients treated with cytarabine at the Department of Hematology at Tokushima University Hospital, 46 patients provided informed consent and were included in this study. We selected 14 tag SNPs located in nine genes that are involved in the cytarabine metabolic pathway; these SNPs were genotyped using the polymerase chain reaction (PCR) restriction fragment length polymorphism (RFLP) technique. Association analyses between adverse reactions to Ara$\mathrm{C}$ therapy and SNPs were performed using logistic regression analysis.

Results: The rs9394992 polymorphism in the SLC29A1 gene and rs3886768 polymorphism in the DCTD gene were associated with the development of rash after Ara-C therapy. The rs7277 polymorphism in the DCTD gene was associated with fever, and the rs16945930 polymorphism in the ABCC11 gene was associated with sore throat.

Conclusions: Our findings suggest that SNPs in the Ara-C metabolic genes influence the development of adverse reactions to Ara-C, and the results suggest that these genes can be predictive of adverse reactions to Ara-C therapy.
\end{abstract}

\section{Keywords:}

cytarabine, single nucleotide polymorphisms, hematopoietic tumor, adverse drug reaction

\footnotetext{
*Corresponding author: Youichi Sato, youichi.sato@ tokushima-u.ac.jp

Article history: Received 9 April 2021, Received in revised form 24 May 2021, Accepted 23 June 2021

https://doi.org/10.46459/pmu.2021002

Copyright (C) 2021 International Society of Personalized Medicine
} 


\section{Introduction}

Hematopoietic tumors are derived from hematopoietic stem cells of the bone marrow that form blood cells. In the past, morphological classification was used, but recently, it has been widely classified as myeloproliferative neoplasms, myeloid/lymphoid neoplasms with eosinophilia and gene rearrangement, myelodysplastic/myeloproliferative neoplasms, myelodysplastic syndromes, myeloid neoplasms with germline predisposition, and acute myeloid leukemia and related precursor neoplasms owing to advances in the elucidation of molecular pathology [1].

Cytarabine arabinoside (Ara-C) is an anti-cancer drug mainly used for the treatment of acute leukemia and exerts anti-tumor activity by inhibiting DNA synthesis. Ara-C was first synthesized in 1559 by Walwick [2]. In 1965, Ara-C was shown to have a significant anti-leukemic effect on acute leukemia. The use of Ara-C was approved in the United States in 1969 and in Japan in 1971. The intracellular transport and metabolic pathways associated with Ara-C are shown in Figure 1. Ara-C is taken up intracellularly by solute carrier family 29 member 1 (SLC29A1) and subsequently phosphorylated by deoxycytidine kinase (DCK), cytidine/uridine monophosphate kinase 1 (CMPK1), and NME/NM23 nucleoside diphosphate kinase 1 (NME1) to produce the cytotoxic component Ara-C triphosphate (AraCTP). Ara-CTP inhibits DNA synthesis and exerts antitumor effects. Ara-C is taken up by cells and is metabolized by cytidine deaminase (CDA) to the inactivated form, Ara uracil (Ara-U). Ara-C is phosphorylated to Ara-C monophosphate (Ara-CMP) by DCK, dephosphorylated by the 5'nucleotidase cytosolic II (NT5C2), and is subsequently inactivated by dCMP deaminase (DCTD). Ara-CMP is exported to the extracellular space by ATP-binding cassette subfamily C member 11 (ABCC11). Ara-CTP is degraded to Ara-C by SAM and HD domains containing deoxynucleoside triphosphate triphosphohydrolase 1 (SAMHD1) [3-5].

Low doses of Ara-C are primarily used for the treatment of acute leukemia, including acute cases of erythroleukemia and chronic myelogenous leukemia. In contrast, high doses of Ara-C are indicated for relapsed or refractory acute leukemia (including myeloid leukemia and acute lymphocytic leukemia) and malignant lymphoma. However, for acute lymphocytic leukemia and malignant lymphoma, the use of Ara$\mathrm{C}$ is limited to combine use with other antineoplastic agents. Ara-C therapy is associated with serious adverse drug reactions, such as hematologic disorders related to bone marrow suppression, anaphylactic shock, and digestive tract disorders. In addition, Ara-C can cause adverse drug reactions, such as digestive disorders, renal dysfunction, skin disorders, neuropsychiatric disorders, fever, and conjunctivitis.

As described above, many adverse drug reactions to Ara$\mathrm{C}$ therapy are known, and some individuals can develop serious adverse drug reactions to Ara-C. Adverse drug reactions reduce the patient's quality of life; therefore, it is necessary to predict the adverse drug reactions in advance and

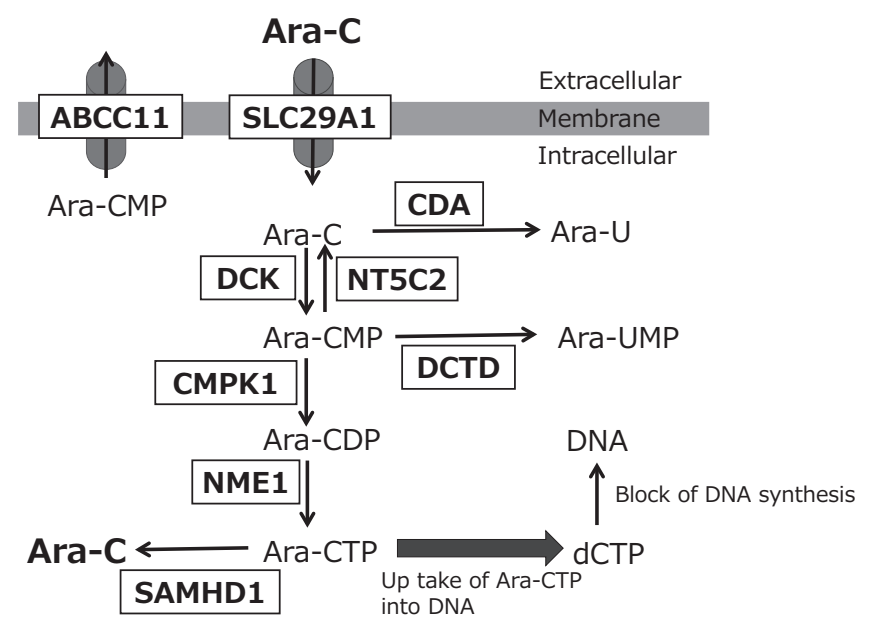

Figure 1. Metabolic pathway of cytarabine.

provide tailored drug therapy for individual patients. It is important to clarify the genetic factors that affect the development of adverse drug reactions as predictors of adverse drug reactions. In the present study, we focused on Ara-C metabolic genes and performed an association analysis between tag SNPs in these genes and adverse reactions to AraC therapy.

\section{Materials and methods}

\section{Patients}

The present study included 46 patients admitted to the Hematology Department at Tokushima University Hospital from January 2015 to December 2019 and who received Ara-C therapy. This study was approved by the Human Genome, Genetic Analysis Research Ethics Committees of Tokushima University (approval numbers: R1-12) and the Clinical Research Ethics Committee of the Tokushima University Hospital (approval numbers: 2425-4). All participants provided written informed consent.

\section{SNP selection and genotyping}

Nine Ara-C metabolic genes, SLC29A1, DCK, CMPK1, NME1, CDA, NT5C2, DCTD, ABCC11, and SAMHD1, were included in this study. We selected 14 tag SNPs in these nine genes (SLC29A1: rs693955, rs9394992, rs324148, and rs760370; DCK: rs1268166; CMPK1: rs3088062; NME1; rs 2159359; CDA; rs558946 and rs517483; NT5C2: rs7920251; DCTD: rs3886768 and rs7277; $A B C C 11$ : rs16945930; and SAMHD1: rs6030200) using the SNPinfo web server [https://snpinfo.niehs.nih.gov/snpinfo/snptag.html] [6] according to the following selection criteria: strong linkage disequilibrium (LD) $\left(r^{2}>0.8\right)$, minor allele frequency $>0.05$, and a minimum of two SNPs tagged each in the Japanese (JPT) population.

Genomic DNA was extracted from saliva samples collected from the patients using Oragene OG-500 saliva collection kits (DNA Genotek Inc., Canada). The rs693955 and 
rs9394992 SNPs were genotyped by Sanger sequencing because they do not contain restriction sites; the other SNPs were genotyped by PCR-RFLP. The DNA from each patient was amplified using EmeraldAmp MAX PCR Master Mix (TaKaRa Bio Inc., Otsu, Japan) under the following PCR cycling conditions: initial denaturation at $94{ }^{\circ} \mathrm{C}$ for $3 \mathrm{~min}$; 30 cycles of $94{ }^{\circ} \mathrm{C}$ for $30 \mathrm{~s}$, annealing temperature for $30 \mathrm{~s}$, and $72{ }^{\circ} \mathrm{C}$ for $1 \mathrm{~min}$; and final extension for $3 \mathrm{~min}$ at 72 ${ }^{\circ} \mathrm{C}$. The resulting PCR products were digested at $37{ }^{\circ} \mathrm{C}$ for $3 \mathrm{~h}$ using the restriction enzymes (New England Biolabs Japan Inc., Tokyo, Japan), as listed in Supplementary Table S 1. The digested products were separated using $2.5 \%$ agarose gel electrophoresis. The fragment sizes listed in Supplementary Table S1 were used for allele identification of the gels.

\section{Statistical analysis}

The associations between adverse reactions to Ara- $\mathrm{C}$ therapy and the SNPs in Ara-C metabolic genes were assessed using logistic regression analysis, and the odds ratios (ORs) and their 95\% confidence intervals (CIs) were calculated. All statistical analyses were performed using $\mathrm{R}$ version 3.5.0 (The R Project for Statistical Computing [http://www.r-proje ct.org]). Statistical significance was set at $\mathrm{p}<0.05$. Significant expression quantitative trait loci (eQTLs) harboring SNPs were searched using the GTEx Portal database (http:// www.gtexportal.org/home/) [7].

\section{Results}

The clinical features of the 46 patients who received Ara$\mathrm{C}$ therapy are presented in Table 1 . The cohort comprised 24 men and 22 women, with an average age of 58.2 years. Among the 46 patients, 21 had acute myeloid leukemia, eight had diffuse large B-cell lymphoma, three had myelodysplastic syndrome, two had acute promyelocytic leukemia, two had follicular lymphoma, two had Burkitt lymphoma, and eight had other diseases (including acute myelomonocytic leukemia, intravascular large B-cell lymphoma, extranodal NK/T cell lymphoma, nasal type, mantle cell lymphoma, peripheral T-cell lymphoma, B-cell lymphoma nontypeable type, granulocyte sarcoma, and Philadelphia chromosome-positive acute lymphoblastic leukemia). In total, 25 and 21 patients were treated with low-dose and highdose Ara-C, respectively. All patients developed grade 4 bone marrow suppression. Therefore, we could not include bone marrow suppression in this study. In addition to bone marrow suppression, the most frequent adverse reactions to Ara-C (including suspected adverse reactions) were digestive disorders $(n=27)$, followed by rashes $(n=23)$, taste disorders $(n=19)$, fever $(n=18)$, mucosal damage $(n=13)$, sore throat $(\mathrm{n}=12)$, hepatic dysfunction $(\mathrm{n}=8)$, anorexia ( $=8)$, eye symptoms $(\mathrm{n}=6)$, hand-and-foot syndrome $(\mathrm{n}=$ $4)$, and headache $(n=3)$. Fever and hepatic dysfunction developed in the late phase, and other adverse drug reactions developed regardless of the early or late phase of therapy.

The genotype and allele frequencies of the 14 SNPs from
Table 1. Characteristic of patients

\begin{tabular}{|c|c|}
\hline \multicolumn{2}{|l|}{ Characteristic } \\
\hline Male - no. & 24 \\
\hline Female — no. & 22 \\
\hline Age - yr $($ mean $\pm S D)$ & $58.2 \pm 11.0$ \\
\hline Age $<50 \mathrm{yr}-$ no. & 8 \\
\hline Age $50-69$ yr - no. & 36 \\
\hline Age $\geq 70 \mathrm{yr}-$ no. & 2 \\
\hline Body mass index $-\mathrm{kg} / \mathrm{m}^{2}($ mean $\pm \mathrm{SD})$ & $22.4 \pm 3.7$ \\
\hline \multicolumn{2}{|l|}{ Diagnosis — no. } \\
\hline Acute myeloid leukemia & 21 \\
\hline Diffuse large B-cell lymphoma & 8 \\
\hline Myelodysplastic syndrome & 3 \\
\hline Acute promyelocytic leukemia & 2 \\
\hline Follicular lymphoma & 2 \\
\hline Burkitt lymphoma & 2 \\
\hline Others & 8 \\
\hline \multicolumn{2}{|l|}{ Dose - no. } \\
\hline Low dose & 25 \\
\hline High dose & 21 \\
\hline \multicolumn{2}{|l|}{ Adverse drug reaction - no. } \\
\hline Digestive disorders & 27 \\
\hline Rash & 23 \\
\hline Taste disorders & 19 \\
\hline Fever & 18 \\
\hline Mucosal damage & 13 \\
\hline Sore throat & 12 \\
\hline Hepatic dysfunction & 8 \\
\hline Anorexia & 8 \\
\hline Eye symptom & 6 \\
\hline Hand-and-foot syndrome & 4 \\
\hline Headache & 3 \\
\hline
\end{tabular}

Others of diagnoses include acute myelomonocytic leukemia, intravascular large B-cell lymphoma, extranodal NK/ $\mathrm{T}$ cell lymphoma, nasal type, mantle cell lymphoma, peripheral T-cell lymphoma, B-cell lymphoma nontypeable type, granulocyte sarcoma and Philadelphia chromosome positive-acute lymphoblastic leukemia.

the 46 patients are summarized in Table 2. To determine whether the 14 SNPs were associated with adverse reactions to Ara-C therapy, we conducted an association analysis with logistic regression analysis using three comparative genetic models (additive, recessive, or dominant). Among the adverse reactions to Ara-C therapy, we conducted an association analysis on cases identified in $\geq 10$ patients. We identified SNPs that were associated with rashes, fever, or sore throat. The results of the association analysis between rashes, fever, or sore throat and the 14 SNPs are shown in Table 3. The rs9394992 polymorphism in the SLC29A1 gene was associated with the development of rashes in response to Ara-C therapy in the additive model $(\mathrm{OR}=0.31,95 \% \mathrm{CI}$ $=0.11-0.84, P=0.022$ ), and the rs3886768 polymorphism at the DCTD gene was associated with the development of rashes in response to Ara-C therapy in the additive model $(\mathrm{OR}=0.32,95 \% \mathrm{CI}=0.11-0.95, P=0.040)$ and dominant model $(\mathrm{OR}=0.21,95 \% \mathrm{CI}=0.06-0.78, P=0.019)$. The rs 
Table 2. Genotype and allele frequencies of 14 SNPs in the 46 subjects.

\begin{tabular}{lllll}
\hline SNP & Gene & Allele $^{\mathrm{a}}$ & Genotypes $^{\mathrm{b}}$ & MAF $^{\mathrm{c}}$ \\
\hline rs693955 & SLC29A1 & T/G & $0 / 28 / 18$ & 0.304 \\
rs9394992 & SLC29A1 & T/C & $5 / 19 / 22$ & 0.315 \\
rs324148 & SLC29A1 & T/C & $1 / 18 / 27$ & 0.217 \\
rs760370 & SLC29A1 & G/A & $3 / 11 / 32$ & 0.185 \\
rs1268166 & DCK & G/A & $0 / 16 / 30$ & 0.174 \\
rs3088062 & CMPK1 & T/C & $7 / 21 / 18$ & 0.380 \\
rs2159359 & NME1 & A/C & $1 / 20 / 25$ & 0.239 \\
rs558946 & CDA & C/A & $2 / 20 / 24$ & 0.261 \\
rs517483 & CDA & G/A & $0 / 21 / 25$ & 0.228 \\
rs7920251 & NT5C & T/C & $5 / 22 / 19$ & 0.348 \\
rs3886768 & DCTD & T/C & $3 / 15 / 28$ & 0.228 \\
rs7277 & DCTD & A/G & $7 / 17 / 22$ & 0.337 \\
rs16945930 & ABCC11 & T/C & $4 / 18 / 24$ & 0.283 \\
rs6030200 & SAMHD1 & A/G & $13 / 16 / 17$ & 0.457 \\
\hline
\end{tabular}

${ }^{\mathrm{a}}$ Allele indicates minor/major allele

${ }^{\mathrm{b}}$ Genotypes indicate minor homozygote/heterozygote/major homozygote

${ }^{\mathrm{c}} \mathrm{MAF}$ indicates the minor allele frequency

7277 polymorphism in the DCTD gene was associated with the development of fever in the dominant model $(\mathrm{OR}=$ $0.28,95 \%$ CI $=0.08-0.97, P=0.044)$. The $r 16945930$ polymorphism in the $A B C C 11$ gene was associated with the development of sore throat in the additive model $(\mathrm{OR}=$ $3.06,95 \% \mathrm{CI}=1.06-8.87, P=0.039)$ and recessive models $(\mathrm{OR}=11.00,95 \% \mathrm{CI}=1.02-119.00, P=0.048)$.

\section{Discussion}

In the present study, several SNPs in Ara-C metabolic genes were found to be associated with rashes, fever, or sore throat. The rs9394992 polymorphism, which is associated with rash development after Ara-C therapy, is located in the intron of the SLC29A1 gene. Therefore, the mechanism by which the rs9394992 SNP affects the function of SLC29A1 is unknown. We then searched for eQTL to investigate the relationship between the expression levels of the rs9394992 SNP and SLC29A1. However, no significant eQTLs were detected. In a previous study, patients harboring the CC genotype of rs9394992 had a longer survival time after receiving Ara-C therapy [8]. Patients harboring the CC genotype of rs9394992 appeared to be more responsive to Ara-C therapy than patients with other genotypes (CT or TT). In addition, patients with the $\mathrm{CC}$ genotype are assumed to have higher levels of Ara-CTP, which is a component of DNA synthesis inhibition, than patients with other genotypes (CT or TT). The above findings suggest that patients with the $\mathrm{CC}$ genotype have a higher intracellular uptake of Ara-C. In this study, the addition of the minor allele $\mathrm{T}$ significantly reduced the development of rashes in response to Ara-C therapy. Patients harboring the $\mathrm{T}$ allele had a lower uptake of Ara-C, which could have reduced the development of rashes. The rs3886768 SNP in the DCTD gene was also associated with the development of rashes in the additive and dominant models. The addition of the minor allele $\mathrm{T}$ to the model significantly reduced development of rashes, and patients with the $\mathrm{T}$ allele showed significantly reduced development of rash compared to patients without the $\mathrm{T}$ allele. The rs3886768 polymorphism is located in the intron of the DCTD gene. Based on the results of searching for eQTLs with rs3886768, the effect of addition of the minor allele to the model was found to significantly upregulate DCTD expression levels in the skin of both sun-exposed and unexposed patients (Supplementary Figure S1A, B). In patients harboring the minor allele, DCTC expression levels were upregulated, and Ara-CMP was metabolized to its inactivated form, Ara-UMP. In addition, the observed reduction in Ara-CMP levels corresponded to a reduction in the levels of cytotoxic Ara-CTP. Reduction in the levels of Ara-CTP, a cytotoxic metabolite of Ara-C, reduced cell damage in the skin, and decreased the development of rashes.

The rs7277 polymorphism in the DCTD gene was found to be significantly associated with the development of fever after Ara-C therapy in the dominant model. The development of fever in patients harboring the minor allele was significantly lower than that in patients without the minor allele. The rs7277 polymorphism is located in the 3' untranslated region (UTR) of DCTD. The results of searching for eQTLs with rs7277 showed that the addition of the minor allele was not known in the bone marrow, but it significantly increased DCTD expression levels in the skin and esophagus mucosa. (Supplementary Figure S1C, D). An increase in DCTD expression levels enhances the metabolism of Ara-CMP to Ara-UMP. Relative levels of Ara-CTP, which is the active ingredient, were decreased, immune response was alleviated, and was accompanied by reduced development of fever.

The rs16945930 polymorphism in the ABCC11 gene was significantly associated with the development of sore throat in response to Ara-C therapy in the additive and recessive models. In other words, the addition of the minor allele significantly reduced the development of rashes. The risk of sore throat development was significantly higher in patients without the major allele than in patients harboring the major allele. The rs16945930 polymorphism is a non-synonymous SNP that causes the substitution of valine with isoleucine. We then predicted the effect of rs16945930 on ABCC11 function using the in-silico SNP prediction algorithm PolyPhen-2 [9]. The prediction classified rs16945930 as "probably damaging," with a score of 0.985 . ABCC11 is ubiquitously expressed in all tissues. Functional damage to ABCC11 impairs extracellular export of Ara-CMP. Levels of Ara-CTP, which is cytotoxic, were found to be relatively increased, thereby causing damage to the pharynx and promoting the development of sore throat.

\section{Conclusions}

In conclusion, although the detailed mechanism is unknown, SNPs in genes involved in Ara-C metabolic path- 
Table 3. Associations derived from different comparative genetic models between 14 SNPs and developments of rashes, fever, and sore throat in response to cytarabine therapy.

\begin{tabular}{|c|c|c|c|c|c|c|c|}
\hline \multicolumn{2}{|l|}{ SNP } & \multicolumn{2}{|l|}{ Rash } & \multicolumn{2}{|l|}{ Fever } & \multicolumn{2}{|l|}{ Sore throat } \\
\hline Gene & Model & OR $(95 \% \mathrm{CI})$ & $\mathrm{P}$ & OR $(95 \% \mathrm{CI})$ & $\mathrm{P}$ & OR $(95 \% \mathrm{CI})$ & $\mathrm{P}$ \\
\hline rs693955 & Add & $3.09(0.90-10.70)$ & 0.074 & $1.50(0.44-5.15)$ & 0.52 & $2.37(0.54-10.30)$ & 0.25 \\
\hline SLC29A1 & Dom & $3.09(0.90-10.70)$ & 0.074 & $1.50(0.44-5.15)$ & 0.52 & $2.37(0.54-10.30)$ & 0.25 \\
\hline rs9394992 & Add & $0.31(0.11-0.84)$ & 0.022 & $1.40(0.58-3.37)$ & 0.46 & $0.49(0.16-1.49)$ & 0.21 \\
\hline \multirow[t]{2}{*}{$S L C 29 A 1$} & Dom & $0.34(0.10-1.14)$ & 0.080 & $1.25(0.38-4.10)$ & 0.71 & $0.56(0.15-2.14)$ & 0.40 \\
\hline & $\operatorname{Rec}$ & NA & NA & $2.60(0.39-17.40)$ & 0.32 & NA & NA \\
\hline rs324148 & Add & $1.85(0.61-5.58)$ & 0.28 & $0.77(0.25-2.35)$ & 0.64 & $0.92(0.27-3.15)$ & 0.89 \\
\hline SLC29A1 & Dom & $1.72(0.53-5.62)$ & 0.37 & $0.85(0.25-2.84)$ & 0.79 & $1.02(0.27-3.88)$ & 0.98 \\
\hline rs 760370 & Add & $0.70(0.26-1.85)$ & 0.47 & $1.79(0.67-4.80)$ & 0.25 & $0.60(0.17-2.11)$ & 0.43 \\
\hline \multirow[t]{2}{*}{ SLC29A1 } & Dom & $1.00(0.29-3.51)$ & 1.00 & $1.91(0.53-6.84)$ & 0.32 & $0.70(0.16-3.10)$ & 0.64 \\
\hline & $\operatorname{Rec}$ & NA & NA & $3.37(0.28-40.30)$ & 0.34 & NA & NA \\
\hline rs1268166 & Add & $1.00(0.297-3.37)$ & 1.00 & $0.38(0.10-1.45)$ & 0.16 & $0.29(0.05-1.51)$ & 0.14 \\
\hline$D C K$ & Dom & $1.00(0.297-3.37)$ & 1.00 & $0.38(0.10-1.45)$ & 0.16 & $0.29(0.05-1.51)$ & 0.14 \\
\hline rs3088062 & Add & $1.31(0.57-3.02)$ & 0.53 & $2.31(0.93-5.72)$ & 0.070 & $1.22(0.48-3.12)$ & 0.68 \\
\hline \multirow[t]{2}{*}{ CMPK1 } & Dom & $1.00(0.31-3.27)$ & 1.00 & $3.50(0.92-13.30)$ & 0.066 & $2.37(0.54-10.30)$ & 0.25 \\
\hline & $\operatorname{Rec}$ & $2.92(0.50-16.9)$ & 0.23 & $2.38(0.47-12.20)$ & 0.30 & $0.42(0.05-3.94)$ & 0.45 \\
\hline rs 2159359 & Add & $1.35(0.46-3.95)$ & 0.59 & $1.54(0.52-4.61)$ & 0.44 & $0.75(0.22-2.60)$ & 0.65 \\
\hline$N M E 1$ & Dom & $1.19(0.37-3.81)$ & 0.77 & $1.33(0.41-4.38)$ & 0.64 & $0.50(0.13-1.98)$ & 0.32 \\
\hline rs558946 & Add & $1.00(0.37-2.71)$ & NA & $1.18(0.43-3.26)$ & 0.75 & $0.64(0.19-2.12)$ & 0.47 \\
\hline \multirow[t]{2}{*}{$C D A$} & Dom & $1.42(0.44-4.53)$ & 0.56 & $1.15(0.35-3.78)$ & 0.81 & $0.44(0.11-1.76)$ & 0.25 \\
\hline & $\operatorname{Rec}$ & NA & NA & $1.59(0.09-27.10)$ & 0.75 & $3.00(0.17-52.10)$ & 0.45 \\
\hline rs 517483 & Add & $2.44(0.74-8.01)$ & 0.14 & $0.92(0.28-3.03)$ & 0.90 & $1.27(0.34-4.74)$ & 0.73 \\
\hline$C D A$ & Dom & $2.44(0.74-8.01)$ & 0.14 & $0.92(0.28-3.03)$ & 0.90 & $1.27(0.34-4.74)$ & 0.73 \\
\hline rs7920251 & Add & $0.82(0.34-1.98)$ & 0.65 & $2.13(0.83-5.51)$ & 0.12 & $2.00(0.72-5.55)$ & 0.18 \\
\hline \multirow[t]{2}{*}{ NT5C2 } & Dom & $0.84(0.26-2.71)$ & 0.77 & $1.73(0.51-5.93)$ & 0.38 & $1.58(0.40-6.26)$ & 0.52 \\
\hline & $\operatorname{Rec}$ & $0.64(0.10-4.21)$ & 0.64 & $7.71(0.79-75.80)$ & 0.080 & $5.33(0.77-37.00)$ & 0.090 \\
\hline rs3886768 & Add & $0.32(0.11-0.95)$ & 0.040 & $0.57(0.20-1.60)$ & 0.28 & $0.62(0.19-2.01)$ & 0.42 \\
\hline \multirow[t]{2}{*}{$D C T D$} & Dom & $0.21(0.06-0.78)$ & 0.019 & $0.67(0.19-2.29)$ & 0.52 & $0.71(0.18-2.84)$ & 0.63 \\
\hline & $\operatorname{Rec}$ & $0.45(0.04-5.66)$ & 0.56 & NA & NA & NA & NA \\
\hline rs7277 & Add & $1.09(0.49-2.42)$ & 0.84 & $0.46(0.18-1.14)$ & 0.093 & $0.47(0.17-1.35)$ & 0.16 \\
\hline \multirow[t]{2}{*}{$D C T D$} & Dom & $1.00(0.31-3.18)$ & 1.00 & $0.28(0.08-0.97)$ & 0.044 & $0.56(0.15-2.14)$ & 0.40 \\
\hline & $\operatorname{Rec}$ & $1.40(0.28-7.12)$ & 0.68 & $0.58(0.10-3.34)$ & 0.54 & NA & NA \\
\hline rs16945930 & Add & $2.41(0.91-6.42)$ & 0.078 & $1.48(0.60-3.70)$ & 0.40 & $3.06(1.06-8.87)$ & 0.039 \\
\hline \multirow[t]{2}{*}{$A B C C 11$} & Dom & $2.92(0.88-9.67)$ & 0.080 & $1.15(0.35-3.78)$ & 0.81 & $2.86(0.72-11.40)$ & 0.14 \\
\hline & $\operatorname{Rec}$ & $3.30(0.32-34.40)$ & 0.32 & $5.40(0.52-56.60)$ & 0.16 & $11.00(1.02-119.00)$ & 0.048 \\
\hline rs6030200 & Add & $1.00(0.49-2.05)$ & 1.00 & $0.94(0.45-1.97)$ & 0.87 & $1.01(0.44-2.29)$ & 0.99 \\
\hline \multirow[t]{2}{*}{ SAMHD1 } & Dom & $1.21(0.36-4.00)$ & 0.76 & $1.29(0.38-4.47)$ & 0.68 & $0.76(0.20-2.93)$ & 0.70 \\
\hline & $\operatorname{Rec}$ & $0.81(0.22-2.92)$ & 0.74 & $0.60(0.15-2.36)$ & 0.47 & $1.39(0.34-5.76)$ & 0.65 \\
\hline
\end{tabular}

Data are shown as odds ratio (OR), $95 \%$ confidence interval (CI) and $\mathrm{P}$ value.

Bold numbers indicate $\mathrm{P}$ value $<0.05$.

The results of recessive model of rs693955, rs324148, rs1268166, rs2159359 and rs517483 are not shown because the results for three adverse drug reaction were not available.

Add, log-additive model (risk allele, minor allele)

Dom, dominant model (minor homozygote + heterozygote vs. major homozygote)

Rec, recessive model (minor homozygote vs. heterozygote + major homozygote)

NA, not available

ways affect drug reactions to Ara-C therapy. These findings are expected to serve as the basis for the accurate prediction of adverse reactions to cytarabine therapy.

\section{Conflicts of interest}

The authors have no conflicts of interest to declare.

\section{Acknowledgements}

We thank Prof. Aiko Yamauchi of Tokushima University for her useful suggestions and discussions. We would like to thank Editage (www.editage.com) for English language editing. 


\section{References}

[1] Swerdlow SH, Campo E, Harris NL, et al, editors. WHO Classification of Tumours of Haematopoietic and Lymphoid Tissues, Revised 4th ed. Lyon, France: IARC; 2017.

[2] Sneader W. Drug discovery: a history. New York: Wiley; 2005, p. 258 doi: 10.1002/0470015535.

[3] Amaki J, Onizuka M, Ohmachi K, et al. Single nucleotide polymorphisms of cytarabine metabolic genes influence clinical outcome in acute myeloid leukemia patients receiving high-dose cytarabine therapy. Int $\mathrm{J}$ Hematol 2015;101:543-53 doi: 10.1007/s 12185-015-1766-4.

[4] Abraham A, Varatharajan S, Karathedath S, et al. RNA expression of genes involved in cytarabine metabolism and transport predicts cytarabineresponse in acute myeloid leukemia. Pharmacogenomics 2015;16:877-90 doi: 10.2217/pgs.15.44.

[5] Schneider C, Oellerich T, Baldauf HM, et al. SAMHD1 is a biomarker for cytarabine response and a therapeutic target in acute myeloid leukemia. Nat Med 2017;23:250-5 doi: 10.1038/nm.4255.

[6] Xu Z, Taylor JA. SNPinfo: integrating GWAS and candidate gene information into functional SNP selection for genetic association studies. Nucleic Acids Res 2009;37(Web Server issue):W600-5 doi: 10.1093/nar/gkp290.

[7] GTEx Consortium. The Genotype-Tissue Expression (GTEx) project. Nat Genet 2013;45:580-5 doi: 10.1038/ng.2653.

[8] Wan H, Zhu J, Chen F, et al. SLC29A1 single nucleotide polymorphisms as independent prognostic predictors for survival of patients with acute myeloid leukemia: an in vitro study. J Exp Clin Cancer Res 2014;33:90 doi: 10.1186/s13046-014-0090-9.

[9] Adzhubei I, Jordan DM, Sunyaev SR. Predicting functional effect of human missense mutations using PolyPhen-2. Curr Protoc Hum Genet 2013;Chapter 7:Unit7.20 doi: 10.1002/0471142905.hg0720s 76. 